\title{
Seamless Monitoring of Physiological Information in Daily Life: Retrospectives and Perspectives
}

\author{
Toshiyo TAmura, ${ }^{*}$, Wenxi Chen ${ }^{* *}$
}

\begin{abstract}
This paper reviews endeavors over the past decades to achieve seamless monitoring of various types of physiological information by a variety of high user-affinity approaches applicable to the daily life environment. Developments in academic research and commercialization from the early period are reviewed. The latest outcomes are briefly investigated and roughly categorized into three main models: miniature portable monitors for ambulatory application, functional fabric-based wearable monitors for better comfort, and unobtrusively deployed invisible monitors for optimum usability. Monitors for seamless monitoring of physiological information in the daily life environment differ from conventional devices that are hospital-centered and aimed at short-term use in clinics. Through scrutinizing the current systems and examining their various pros and cons, we identify existing common concerns, provide insight into problem determinants, and suggest research topics for further study. In the near future, we envision that the home will be transformed into an intelligent hub for lifelong healthcare through seamless monitoring of the human body in the daily life environment, which will foster the development of a new discipline "Metrology of Health" or "Healthology" based on a holistic view of health.
\end{abstract}

Keywords: Aging in place, seamless monitoring, unobtrusive monitoring, invisible monitoring, ubiquitous monitoring, wearable monitoring, portable monitoring, home healthcare, Metrology of Health, Healthology.

Adv Biomed Eng. 4: pp. 86-95, 2015.

\section{Introduction}

With the advent of information and communication technology (ICT), and its pervasive application in medical and healthcare domains, life expectancy at birth in 35 countries worldwide has extended into the 80s for both females and males [1]. During the first half of the 21 st century, the proportion of the population aged 60 years and over will double from about $11 \%$ to $22 \%$, representing an increase in number from 605 million to 2 billion [2]. One of the greatest concerns in the aged population is the increase in chronic conditions that account for $60 \%$ of total deaths [3]. The decline in health due to aging results in a decrease in personal quality of life and poses a huge burden in terms of global financial expenditure. Healthy aging is therefore of paramount importance.

Global action for healthy aging has been undertaken at different levels. National campaigns for health promotion and disease prevention have been implemented in Australia [4], Canada [5], China [6], the EU [7], Japan [8], Korea [9], the UK [10], USA [11], and many other countries. Such nationwide campaigns aim to promote the health condition of citizens through a total package program from national law to personal daily activities, and are sustained for several decades.

At the institutional level, a community-based social service

Received on July 22, 2014; revised on October 16, 2014 and March 1, 2015; accepted on April 4, 2015.

* Faculty of Biomedical Engineering, Osaka Electro-Communication University, Neyagawa, Japan.

** Biomedical Information Technology Lab., Research Center for Advanced Information Science and Technology, The University of Aizu, Aizu-Wakamatsu, Japan.

\# 18-8 Hatsucho, Neyagawa, Osaka 572-8530, Japan.

E-mail: tamurat@isc.osakac.ac.jp "aging in place" initiative known as NORC-SSP (Naturally Occurring Retirement Communities-Supportive Services Program) in the mid-1980s was designed to promote healthy aging and healthcare management in New York [12]. Since the early 1990s, a government-sponsored municipal project, "Challenge to 100 years of age," was pioneered in West Aizu Village in northern Japan [13]. The fundamental goal is to live longer independently with better quality of life by providing a total care solution to villagers and encouraging them to practice a healthier lifestyle.

To support "aging in place" and "daily health promotion," accurate detection and early warning of changes in health condition are indispensable. Seamless monitoring of physiological information in various living scenarios over long periods without disturbing daily activities can significantly enhance caregivers' ability to deliver evidence-based care. A study on congestive heart failure (CHF) patients showed that use of telehealth technology decreased overall utilization of healthcare resources by $41 \%$; physician office visits decreased by $43 \%$, emergency department visits by $33 \%$, and hospitalizations by $29 \%$ [14].

For seamless monitoring of multifaceted physiological information in the daily life environment, many academic goals have been addressed and a variety of technological problems have been experienced over the past several decades. Research and development have gone through different approaches to enhance several aspects such as miniaturization, comfort, and concealment, to achieve better user affinity in different application scenarios. Miniaturization technology helps implement smaller and lighter portable monitors for ambulatory application. Wearable monitors target pervasive application in daily activities without discomfort. Invisible methods are usually achieved by concealing sensors or transducers in furniture and appliances for indoor applications. Some of these technologies have matured and have been commercialized for use in daily settings, but some have yet to be achieved and further studies are required. 
This paper presents a review of the latest developments and various commercial products, discusses their appealing features and available applications, and outlines some topics for future exploration. Finally, we expect that in the future, the home will become a hub for seamless monitoring of various physiological information for lifelong healthcare.

\section{Current Achievements}

Noninvasive electrocardiography (ECG) was first developed in 1901 by Willem Einthoven [15] who used a string galvanometer that required water-cooling and five operators and weighed some $270 \mathrm{~kg}$. However, the latest ECG devices are pocket-sized, $9 \mathrm{~mm}$ thick, and weigh only $13 \mathrm{~g}$ [16]. Various modalities for ECG measurement have been developed and can fit into our clothing, mobile phones, and indoor furniture such as beds [17-20], chairs [21], bathtubs [22, 23], and even in the shower room [24]. In addition to ECG, increasing amounts of physiological information can be monitored conveniently using a range of modalities based on various engineering principles.

With the development of micro-electromechanical systems (MEMS), integrated circuits (IC), nano, and material technologies, monitoring devices suitable for daily application have evolved along many different lines over the past several decades. Nevertheless, they can be roughly classified into three categories: portable, wearable, and invisible.

Portable monitors have been developed mainly for ambulatory usage. The early stages of development focused on miniaturization, improvement of usability, and prevention of artifacts. Advances in IC and MEMS technologies have made it possible to reduce both their size and weight. These devices can be used to obtain multiple types of physiological information, such as ECG, blood pressure, body temperature, calorie consumption, and physical activity.

Wearable monitors are usually embedded into a garment or accessory, and worn by the user as part of their clothes without the need to carry extra items. They are unobtrusive, comfortable, esthetic, and functional without affecting daily activities. Some common examples are underwear [25, 26], brassiere [27], adhesive plaster [28], shoes [29], hat, eyeglasses [30], belt, watch, bracelet, and hairpin.

Invisible monitors are usually concealed in home utilities such as furniture, electrical appliances, and sanitary equipment. They are noncontact and invisible to the user, and can function in a fully unobtrusive and automatic manner at a fixed indoor site, without requiring any intervention from the user.

As the pervasive use of ICT has provided a common gateway with connection to the Internet, all of these monitors can be linked to a mobile phone platform. Therefore, a network connection can be maintained indoors or outdoors.

The following sections present some examples of these types of equipment.

\subsection{Portable Monitors}

A portable monitor for continuously monitoring ECG was introduced by N.J. Holter more than 50 years ago [31]. It consisted of an 85 -pound $(38.6 \mathrm{~kg}$ ) radio transceiver and could record $24 \mathrm{~h}$ or more of ECG. Despite requiring the same time as recording to interpret the measured ECG, the importance of the Holter portable device was recognized immediately by the medical communi- (a)

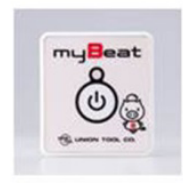

(d)

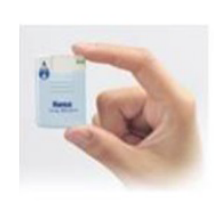

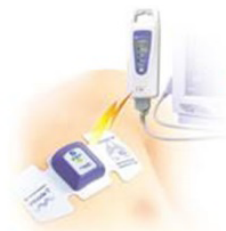

(b)

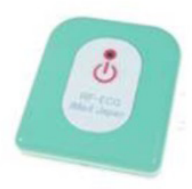

(e)

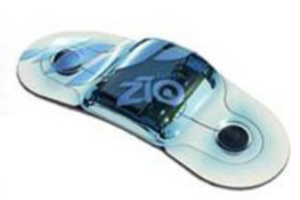

(c)

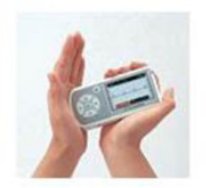

(f)
Fig. 1 Cordless ECG monitors: (a) Cardy 303 pico+, (b) ZU-110P, (c) Zio Patch, (d) myBeat, (e) RF-ECG, (f) eHeart. (All reproduced with permissions).

ty following a large number of clinical findings in arrhythmia, silent myocardial ischemia, sudden death syndrome, and pacemaker follow-up, because such data were difficult to capture during occasional clinic visits due to their transient nature. This led to the widespread acceptance of the device as a routine clinical technique for ECG monitoring without markedly affecting daily activity.

Over the following five decades, a great deal of effort was invested toward achieving better usability, longer recording time, faster interpretation speed, smaller size, lighter weight, higher digitized resolution, more channels, and more functions. As a result, many similar products were developed.

The devices became smaller and lighter and yet more functional. In addition to ECG, these devices can simultaneously monitor multiple types of information such as activity and body temperature. The "Cardy 303 pico+" is waterproof and can be used during bathing, is only $9 \mathrm{~mm}$ thick, and weighs $13 \mathrm{~g}$. A three-axis acceleration sensor is built-in for monitoring gestures and activities, as shown in Fig. 1(a) [16].

Different clinical purposes may require different electrode schemes. One problem with the traditional Holter device is the so-called cord spaghetti syndrome. A pilot study on a cordless ECG monitor aimed at mitigating the spaghetti syndrome and improving usability was conducted in the early 1990s [32]. An electrode pad was adhered directly to the body surface to measure two channels of ECG continuously over $24 \mathrm{~h}$. Measured ECG was transmitted from the pad to a tape recorder real-time and without wire. Several devices such as ZU-110P (Fig. 1(b)) [33] and Zio Patch (Fig. 1(c)) have been commercially available since the early 2000s. The Zio Patch is an adhesive patch-type monitor for continuous ambulatory recording of single-lead ECG. In comparison with conventional Holter monitors that usually function for only 1 day, a pilot study involving 146 cardiac patients indicated that the Zio Patch could remain attached for as long as 14 days and detect arrhythmia more accurately without missing too many events [34-37].

Real-time processing, network connectivity, and more parameters are bundled into a compact size. The "myBeat WHS-2" (Fig. 1(d)) [38] and "RF-ECG" (Fig. 1(e)) [39] measure not only ECG but also body temperature and three-axis acceleration. The 


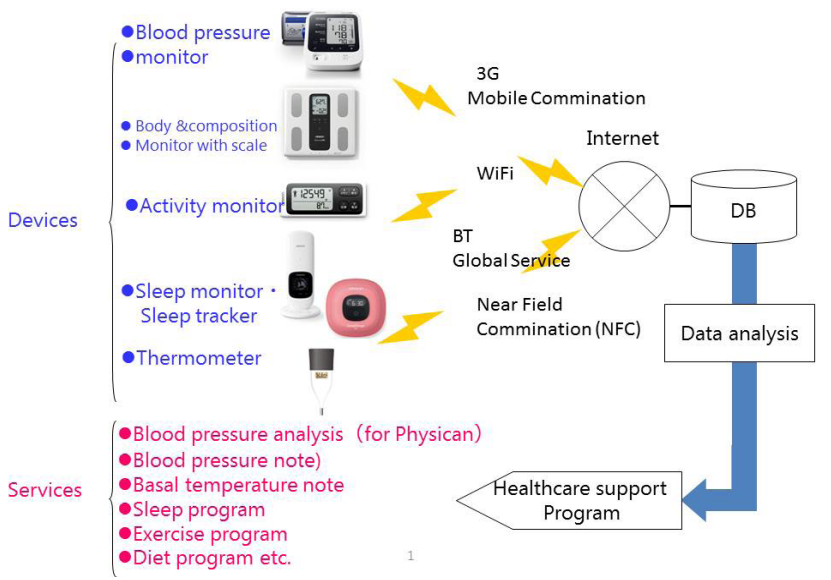

Fig. 2 Home-oriented portable monitors by Omron: (reproduced with permission).

former is $13.7 \mathrm{~g}$ in weight and $10 \mathrm{~mm}$ in thickness, can function for $20 \mathrm{~h}$ using a rechargeable lithium ion button battery, and uses Bluetooth Smart communications for data exchange. Its application programing interface (API) is provided free of charge and is open to partners.

One significant derivative is an event monitor that can promptly capture emergent events such as transient arrhythmia and related symptoms, while reducing the redundancy of data recording through real-time interpretation of the ECG signal, thereby making it possible to reduce the size of the device, similar to a wristwatch [40]. The "eHeart" is a pocket-sized portable ECG recorder that can record 12 events each of 24-s duration, as shown in Fig. 1(f) [41].

In outdoor applications, a portable monitor usually makes use of a mobile phone for data transmission. In indoor applications, a portable monitor often has more flexible options such as Bluetooth, infrared, camera, and near-field communication (NFC) for data transmission to a base station, which relays the data to remote servers.

In addition to ECG, many other types of information such as $\mathrm{SpO}_{2}$, glucose, blood pressure, respiratory functions, breath gas, body temperature, body fat, body weight, and motion have been integrated in a set of home-oriented portable devices. Most of these are directed toward both general wellness as well as chronic conditions such as congestive heart failure, chronic obstructive pulmonary disorder, asthma, hypertension, diabetes, obesity, and sleep disorders. Commercial products include MedStar [42], TeleHealthcare Network [43], Tiered Platforms [44], CardioBlips [45], LifeStar [46], MedicalLINK [47], HealthPlanet [48], Sentry Telemonitor [49], Health Buddy System [50], BeClose [51], e-neighbor system [52], Care Innovations Connect Platform [53], GrandCare Com [54], Philips' Telestation [55] Omron Wellness Link [56] and Sotera Wireless Visi Mobile System [57]. Figure 2 shows a typical example of these systems.

The Visi Mobile includes noninvasive beat-to-beat blood pressure measurement that is either cuff-based or cuffless. Using the cuff module, blood pressure is measured and then the pulse transit time is continuously monitor to estimate the blood pressure [57]. The FDA approved cuffless, non-invasive, continuous blood pressure monitoring in 2013.

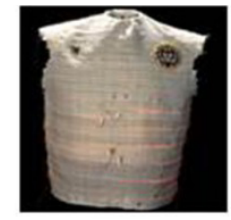

(a)

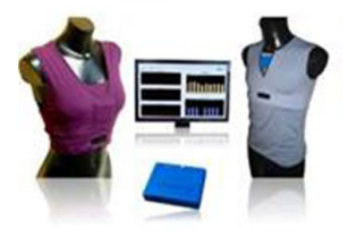

(c)

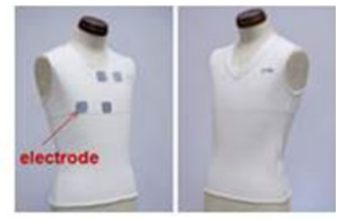

(b)

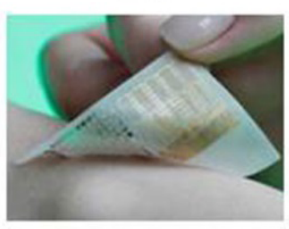

(d)
Fig. 3 Representative wearable monitors (a) Smart Shirt (provided by Sundaresan Jayaraman/Georgia Institute of Technology, reproduced with permission) (b) PEDOT-PSS (left=interior, right=exterior) (provided by NTT), (c) Wearable Wellness System (reproduced with permission), (d) Adhesive flexible plaster (reproduced with permission).

\subsection{Wearable Monitors}

Clothing is a good candidate for a more natural means of seamless monitoring of physiological information indoors or outdoors. Articles typically worn in daily life, such as accessories, hats, wrist watches, shirts, shoes, finger rings, bracelets, brassieres, barrettes, and belts can be used.

Sensing technology can be incorporated in traditional fabrics, or the textile itself can be made functional [58].

In the mid-1990s, the "Smart Shirt," which integrated conductive fibers and a host of vital sign sensors, was developed to monitor the wearer's heart rate, ECG, respiration, temperature, and voice [25], as shown in Fig. 3(a).

Most functional textiles are made primarily of metallic or optical fibers that are fragile and uncomfortable to wear, and they also corrode and are non-washable. Alternatively, coating silk fibers with the conductive polymer PEDOT-PSS improves tensile strength and functionality without any adverse effects on biocompatibility, hydrophilicity, or flexibility [59], as shown in Fig. 3(b). A similar product, the Wearable Wellness System (WWS) that uses yarns with built-in sensors can acquire ECG, respiratory signals, and tri-directional body movements, and extract several parameters such as heart rate, respiration rate, signal quality index, posture, activity, and energy expenditure [26], as shown in Fig. 3(c). The WWS was developed as a part of the EU-funded "MyHeart" project, which aimed at continuous monitoring of vital signs to gain information regarding the health status of an individual in a natural environment [60]. They were designed to be more similar to a piece of clothing and less like a medical device.

By alternately dipping normal cotton yarn into a solution of conductive carbon nanotubes in water and a solution of special sticky polymer in ethanol several times, the yarn becomes highly conductive but remains pliable and soft [61, 62]. Moreover, addition of, for example, an anti-albumin antibody to the carbon nanotube solution results in a reaction with albumin in blood, leading to a significant increase in conductivity of the fabric. Any antibody can be incorporated into the yarn and used to detect other biomolecules and proteins. 


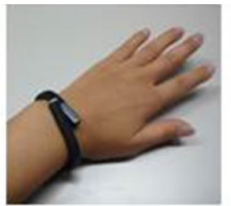

(a)

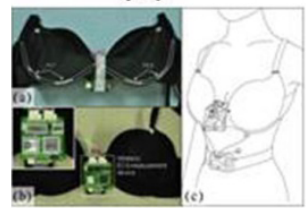

(c)

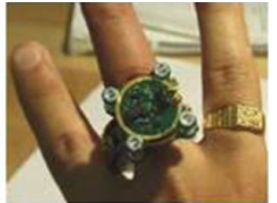

(b)

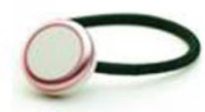

(d)
Fig. 4 Accessories and female items as wearable monitors. (a) Fitbit, (b) finger ring (reproduced with permission), (c) e-brassiere (provided by Kwangsuk Park, reproduced with permission), (d) Karada Fit. (reproduced with permission).

Industrial exploitation of functional textiles is still in its infancy, but the number of technological implementations is increasing. A thorough investigation reviewed many of the latest advances in this area [63]. Clevertex aims to develop a strategic plan for the transformation of traditional textiles and clothing into a knowledge-driven industrial sector by 2015 .

Epidermal electronics can be used to develop flexible and stretchable stamp-sized patches that can be attached to the skin to monitor electrical activity [64]. A flexible patch that integrates nano-scale sensors and drug delivery components onto a stretchable polymer substrate is used not only as a monitor but also as a therapeutic device $[28,65]$. The patch can measure EMG data and perform real-time processing to determine the proper timing for delivery of medicine through the skin, as shown in Fig. 3(d).

Accessories such as watches, bracelets, and finger rings are useful devices for daily monitoring. Figure 4(a) shows a bracelet that serves as an activity and sleep monitor when worn day and night. Multiple measurements such as photoplethysmogram, pulse rate variability, $\mathrm{SpO}_{2}$, and blood pressure can be performed by a finger ring, as shown in Fig. 4(b) [66].

More options are available for women, such as a brassiere with embedded gold nanowires as electrodes for monitoring ECG in daily life [27, 67]. This was further developed to make evidence-based decision to automatically unlock the brassiere in the presence of a lover by real-time processing of heart rate variability, as shown in Fig. 4(c). "Karada Fit MTN-200" is similar in appearance to a hair holder but can measure activity and connect to a mobile phone via NFC [68], as shown in Fig. 4(d).

Movements are often the source of motion artifacts in physiological measurement for ambulatory applications. Various algorithms and instruments have been deliberated to achieve reliable measurement and higher signal quality by removing the movement signals [69]. Nevertheless, one man's trash may be another man's treasure. The movement signal is a useful indicator in evaluating human activity. More than 20 activity monitors are available commercially [70].

When a shoe is embedded with an insole pressure sensor and an accelerometer, as shown in Fig. 5(a), movement can be an important signal for monitoring daily activities, such as energy expenditure and major postures [71]. The "SenseWear" arm-

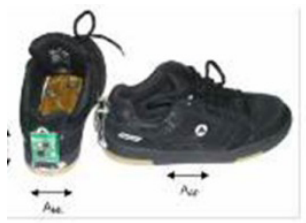

(a)

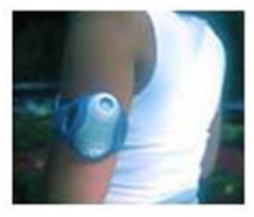

(b)

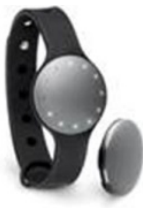

(c)
Fig. 5 Activity monitors. (a) Shoes with insole pressure sensor and accelerometer (reproduced with permission), (b) SenseWear, (c) Misfit Shine (reproduced with permission).

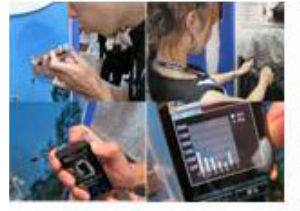

(a)

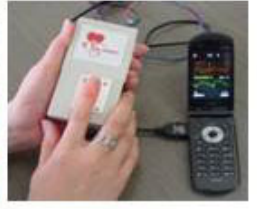

(b)

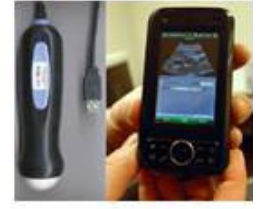

(c)
Fig. 6 Mobile-phone-based monitors. (a) Multiple functions, (b) sphygmomanometer, (c) ultrasound image (Photographs provided by David Kilper/WUSTL, reproduced with permission).

band $[72,73]$ acts as a versatile monitor of daily physical activity and sleep behaviors in a free-living environment, as shown in Fig. 5(b). The "Misfit Shine" is a clasp and can track activities on land and in water, running or swimming, when clipped onto clothing [74], as shown in Fig. 5(c). It weighs less than $10 \mathrm{~g}$ and uses a coin battery that provides power for 4 months of use.

In addition to clothing and accessories, mobile phones are now integrated into our daily life and are becoming indispensable. A mobile phone can be used to monitor falls [75]. A mobile phone may be fitted with integrated sensors for monitoring respiration gas, body fat, activity, calorie consumption, and photoplethysmogram [76], as shown in Fig. 6(a). A mobile phone can also be transformed as a satisfactory platform to serve as a sphygmomanometer [77], as shown in Fig. 6(b), or an ultrasound imaging monitor [78], as shown in Fig. 6(c). The potential of mobile phones in application of healthcare domain remain to be further excavated [79]. The wellness paradigm of mobile phones will facilitate daily healthcare wherever there is mobile network coverage.

Wearable monitors are evolving toward increased comfort, functionality, mobility, affinity, and esthetics, and will finally merge into daily personal items without sharp demarcation.

\subsection{Invisible Monitors}

Although wearable monitors can be used both indoors and outdoors without marked disturbance of daily life, some are in direct contact with the human body, and therefore may be associated with problems such as unpleasant or allergic reactions. When application is limited to indoor use only, many types of physiological information can be monitored by noncontact means, where sensors are concealed and invisible to the user. Various sensors can be embedded into a wide variety of fixed home appliances such as beds, toilets, and bathtubs, or even in moving objects such 


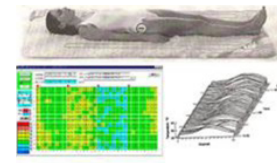

(a)

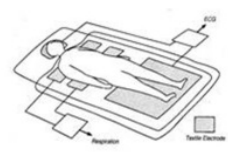

(b)

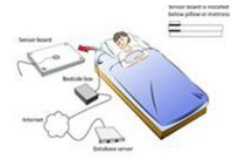

(c)
Fig. 7 Bed monitors for various vital signs. (a) Body temperature distribution map, (reproduced with permission) (b) electrocardiogram and respiratory rate, (c) ballistocardiogram.

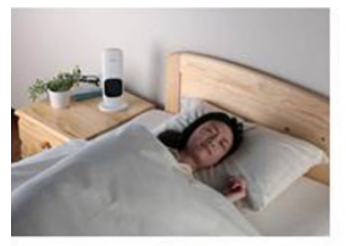

(a)

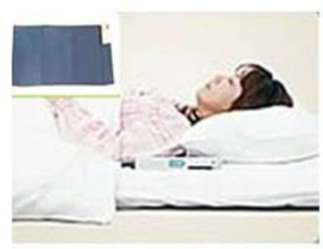

(c)

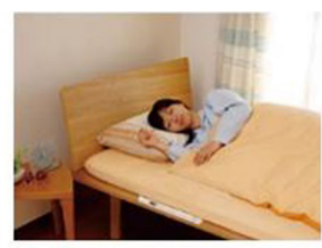

(b)

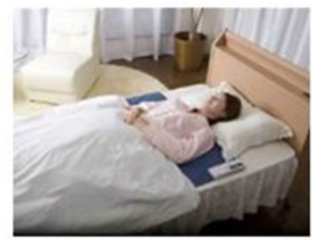

(d)
Fig. 8 Commercial sleep monitors. (a) Sleep Design HSL-101, (b) Installed image of Sleep Scan SL-504, (c) Sleep EYE GD700, (d) Sleep Recorder SD-101 (All reproduced with permissions).

as cars. Over the past three decades, many academic studies have been conducted and some of their outcomes have been developed commercially.

Figure 7 shows bed monitors for various vital signs, such as (a) body temperature mapped by a $16 \times 32$ thermal sensor array [80], (b) ECG by two electroconductive textile sheets at the head and feet [81] and respiratory rate at the thorax and abdomen sheets [82], and (c) ballistocardiogram and respiration by a piezo-electric pressure sensor installed beneath the pillow or the mattress [83].

Many sleep monitors are commercially available as screening tools for sleep apnea and other sleep disorders. These devices usually monitor heart rate, respiration rhythm, and body movement by various engineering principles, and derive sleep parameters such as sleep efficiency, sleep latency, and sleep stage. Figure 8 shows (a) a noncontact monitor based on Doppler radar [84-87], (b) a sensor board filled with water for sensing body pressure/vibrations [88], and (c) a thin sheet with multiple pressure-sensitive cells [89-91].

Various sensors can be embedded into sanitary facilities such as bathtubs and toilets without interfering with their utilization. Figure 9 shows some examples: (a) a bathtub monitor that can measure ECG via stainless steel electrodes on the bathtub wall [23]; (b) the shower head and the ground constitute two electrodes for ECG measurement during shower [92-94]; (c) ECG is measured when the thighs contact two electrodes attached to the toilet seat, and body weight, urinary volume and flow rate, and sugar level are analyzed simultaneously [81,95]; (d) "Thermo

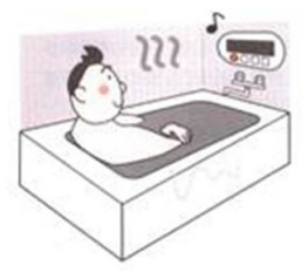

(a)

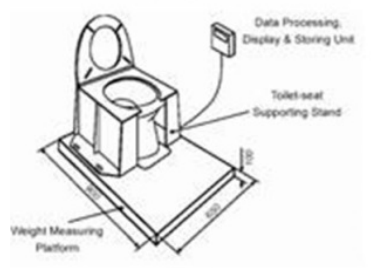

(c)

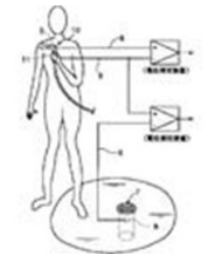

(b)

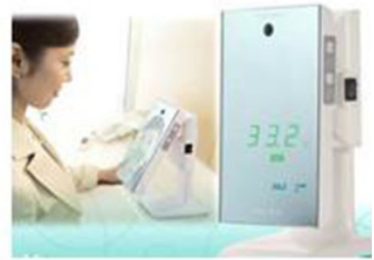

(d)
Fig. 9 Sanitary-facility-based monitors embedded into a (a) bathtub (reproduced with permission), (b) shower, (c) toilet (reproduced with permission), and (d) dressing mirror (reproduced with permission).

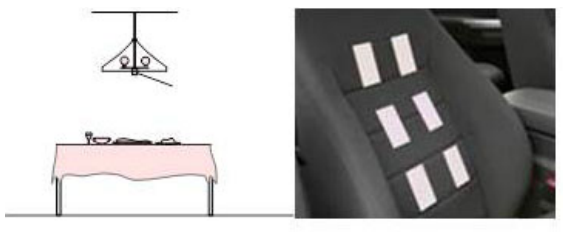

(a) (b)

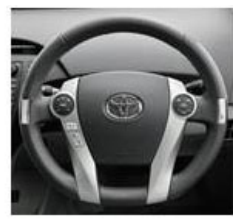

(c)
Fig. 10 Fixed and moving invisible monitors. (a) Dining table and a ceiling lamp with a built-in video camera, (b) car seat, (c) car steering wheel (photographs provided by Denso, reproduced with permission).

Mirror" has an appearance of a cosmetic mirror but has a thermal sensor embedded to measure the temperature of facial skin without physical contact [96].

Many other fixed and movable objects can serve as invisible monitors for daily monitoring. A "magic carpet" embedded with a mesh of optical fibers detects deflected light patterns and is able to estimate walking ability of the elderly for early detection of erratic movements and mobility problems [97].

Figure 10 shows (a) a dining table and a ceiling lamp with a built-in video camera, (b) a car seat, and (c) a car steering wheel as invisible monitors. The dining table can be used to monitor daily nutrition intake by the video camera integrated within the ceiling lamp above the table [98]. Capacitive electrodes are embedded in a chair [21] or car seat [99] to monitor ECG while sitting. Two pieces of metal electrodes are affixed to the left and right sides of the steering wheel to detect ECG, and a reflective photoelectric sensor is embedded on the right side for photoplethysmography when driving [100].

Many modalities of invisible monitors based on various engineering principles, which can be deployed in furniture indoors or moving vehicles outdoors, are being developed. 


\section{Tasks and Topics for Future Study}

Daily healthcare is a more pressing challenge than ever before in the aging society due to the dominance of chronic diseases in the elderly. To facilitate effective healthcare, studies have aimed to achieve increased data collection rates, more accurate interpretation of physiological significance, less disturbance of daily activity, ease of use, and usability by untrained and unskilled users [101]. Remarkable technical innovations have facilitated seamless monitoring of physiological information, and a wide variety of solutions and products have covered various scenarios in daily life. More options are now available regardless of indoors or outdoors, awake or asleep, sedentary or ambulatory.

Nevertheless, many political, administrative, medical, and technical problems remain to be resolved. A WHO report pointed out that current societal systems do not sufficiently distinguish between acute and chronic care, and patients are seldom provided with a long-term management plan for chronic conditions to ensure the best outcomes. This report suggested that an innovative strategy and action plan at the national level is required. Healthcare should be considered as a long-term investment that will yield national dividends. Countries must change their policies to establish a stepwise framework to offer a flexible and practical approach; for example, by making health insurance applicable to not only diagnosis and treatment but also prevention and health promotion. Such a framework will lead to significant improvements in prevention of chronic disease [102].

Collaboration between the medical field and engineering societies is of paramount importance. Medical doctors and healthcare workers should recognize the current shift in the medical paradigm from irregular clinical visits to home-based daily monitoring, play an active role in promoting health and not merely treating disease, make full use of newly developed devices, attract more attention from other related sectors, and act as a bridge among end-users, developers and policy makers. Engineering societies should supply accurate, convincing, and reliable evidence that seamless monitoring in daily life can reduce medical and health insurance costs. Provided that the importance of seamless monitoring in daily life is acknowledged by all relevant stakeholders from policy makers and end-users to healthcare workers and medical doctors, awareness will be shared and cooperative actions will be taken promptly from the administrative and enterprise levels to the community and individual levels.

To overcome the obstacles to further popularization, several technical aspects must be emphasized, such as how to detect physiological information without interfering with activities of daily living, how to perform in-depth data analysis for comprehensive and accurate interpretation of physiological significance, and how to provide a secure platform for individuals and physicians.

Persistent data collection is indispensable in daily health management because health condition tracking requires data accumulation over a long period. The requirement for minimal disturbance of activities of daily living often limits technical options and leads to issues such as low signal-to-noise ratio (SNR) and poor signal quality. Utilization in the daily environment and by nonprofessional users is frequently a cause of inaccurate measurements, uncontrollable data loss, and interrupted monitoring. Even those aware of the importance of daily monitoring to main- tain a healthy life and sufficiently motivated to monitor their physiological conditions may still hesitate to manipulate a complicated medical device on a daily basis [103]. These problems usually reduce the reliability of deep mining of physiological information.

For persistent data collection in daily scenarios, user-centered affinity with various sensors and measurement sites should be taken into account; innovative modalities should include some common features such as scalable systematic architecture to allow personalized customization, autonomous troubleshooting and automatic recovery from failure, network-based administration for remote assistance, zero maintenance, and fully automated operation to reduce the need for user intervention. An ambitious clinical trial to facilitate health management even in healthy individuals is currently underway, in which a tiny cardiac monitor, approximately one-third the size of a AAA battery, is implanted in the chest over the heart for continuous monitoring up to 3 years [104].

However, issues remain regarding poor signal quality, fragmented information, and data discontinuity due to unavoidable data loss in measurement and transmission over the network. To obtain reliable results from such multifaceted data, appropriate models to estimate the signal quality index (SQI) of the measured data are necessary to establish an optimal threshold for exclusion of lower SQI data. More robust algorithms are required to complete and surrogate inconsistent data due to outliers and artifacts. These will improve the reliability of the analytical outcomes. A series of attempts have been undertaken to deal with the missing RR intervals during daily monitoring, using several statistical models [105-107]. Physiological significance can therefore be interpreted more accurately in terms of identifying the statistical links between dynamic health condition and physiological information, and to provide explicit evidence for health management practice.

Fortunately, Big Data platforms and cloud computing infrastructure are open, scalable, and can be used to store and share huge volumes of information over the network. This facilitates realization of efficient mining of not only physiological information but also psychological and social well-being data that can be collected from the social networking service (SNS) infrastructure, and provides an effective approach to evidence-based health management using a holistic view of health. Insufficient privacy protection due to systematic security vulnerabilities is another major problem when large amounts of personal data are transferred, exchanged, and stored over network infrastructure. Traditional measures such as one time password (OTP), secure sockets layer (SSL), and secure electronic transactions (SET) protocols, as well as redundant and replicate system implementation can guarantee data security and protect privacy. Moreover, identity authentication by automatic identification of personal physiological information is another available security option in biomedical systems.

Charging the battery of monitoring devices during the day is inconvenient for the user. Power solutions are thus critical for maintaining consistent monitoring. Energy harvesting through optical and thermal conversion as well as wireless radio-electromagnetic transmission may provide solutions to these issues [108, 109]. Indoor illumination can provide not only lighting but can also carry information over distances [110]. A conventional home 
can be turned into an intelligent hub for implementation of healthy aging management by embedding a variety of such monitors into the furniture and appliances.

As gathering of data for a short period does not provide significant evidence for quantitative evaluation of health, informed consent should be obtained from the users for their daily involvement over a long period. In contrast to occasional clinic visits, daily monitoring requires constant input from the users either by their involvement in self-management or by provision of essential information to ensure optimal outcomes. Seamless monitoring using the approaches described here has many advantages such as rapid response to emergent situations, less inconvenience to the user, minimal intrusion by concealment, no requirement for sensors to be attached to the body, gender neutrality and one-sizematch-all. Therefore, such approaches are beneficial for elderly persons who are generally unfamiliar with manipulation of technical equipment. This will result in greater acceptance of "aging in place" in elderly healthcare.

\section{Conclusions}

It is an arduous task to select symbolic instances from the huge numbers of publications worldwide. We therefore did not intend to cover all aspects of seamless monitoring in this article. Instead, we have presented an overview of this emerging field through retrospective examination of the achievements over the past several decades, to encourage further collaborations among different professional disciplines and at various administrative levels.

The borders among portable, wearable, and invisible monitors are becoming rather indistinct. One device can be transformed into another when the application scenario changes. A wearable outdoor monitor can turn into an invisible monitor when used indoors. Although many challenges remain, with the maturation of technical and societal atmospheres and the dream of achieving a healthier and longer life through innovation, the prospect of seamless monitoring is a promising emerging field of interdisciplinary research. This is referred to as "Metrology of Health" or "Healthology," which aims to quantify the overall health status in an integrative manner and to untangle the causal connections among longevity and relevant determinants such as pathogenesis and immunity, meteorological and environmental factors, and social behavioral-psychoneurotic interactions, through further exploration.

\section{Acknowledgement}

This work was supported in part by grant-in-aids from the Regional Innovation Strategy Support Program, Ministry of Education, Culture, Sports, Science and Technology, Japan, 2011-2016; and from Strategic Information and Communications R\&D Promotion Programme (SCOPE), Ministry of Internal Affairs and Communication, Japan, 2014-2017; and a grand-in-aid for the "Development of algorithm and software for m-health sensor" for the cooperative project with Samsung R\&D Institute Japan.

We thank Georgia Institute of Technology, Seoul National University, Washington University St Louis, and Denso Corp. and Omron Healthcare Co. Ltd. for their kind permissions to use the relevant photographs in this review.

\section{Conflict of Interest}

The authors declare no conflict of interest.

\section{References}

1. CIA: "The World Factbook," [Online]. Available: https://www. cia.gov/library/publications/the-world-factbook/rankorder/ 2102rank.html [Accessed on February 21, 2014].

2. WHO: "Ageing and Life Course," [Online]. Available: http:// www.who.int/ageing/en/ [Accessed on April 22, 2014].

3. WHO: "WHO Global Report: Chronic Diseases and Health Promotion," [Online]. Available: http://www.who.int/chp/chronic_ disease_report/part1/en/ [Accessed on April 22, 2014].

4. Australian Government: "Health Promotion," [Online]. Available: http://australia.gov.au/topics/health-and-safety/healthpromotion [Accessed on April 22, 2014].

5. Minister of Health: "Health Canada," [Online]. Available: www. hc-sc.gc.ca [Accessed on April 22, 2014].

6. China Health Promotion Foundation, "Personal Health Promotion Program," [Online]. Available: http://www.chinahpf.org. cn/CPItemA.aspx?id=394 [Accessed on April 22, 2014].

7. European Partnership for Action Against Cancer: "Health Promotion and Prevention," [Online]. Available: http://www.epaac.eu/ health-promotion-and-prevention [Accessed on April 22, 2014].

8. "Healthy Japan 21" [Online]. Available: http://www. kenkounippon21.gr.jp/ [Accessed on April 22, 2014].

9. Korea Association of Health Promotion: "Health promotion project," [Online]. Available: http://english.kahp.or.kr/cms/doc. php?tkind $=92 \& 1$ kind $=68$ [Accessed on April 22, 2014].

10. Pharmaceutical Services Negotiating Committee: "Public Health (Promotion of Healthy Lifestyles)," [Online]. Available: http:// psnc.org.uk/services-commissioning/essential-services/ public-health/ [Accessed on April 22, 2014].

11. Centers for Disease Control and Prevention: "Gateway to Health Communication \& Social Marketing Practice," [Online]. Available: http://www.cdc.gov/healthcommunication/campaigns/ index.html [Accessed on April 22, 2014].

12. "NORCs - An Aging in Place Initiative" [Online]. Available: http://www.norcs.org/ [Accessed on April 22, 2014].

13. West Aizu Village, Challenge to 100 Years of Age - The Making of a Healthy Village through a Total Care Solution, Fukushima: Zaikai21 Publishing House, 2003.

14. Lehmann C, Nancy MN, Giacini J: Impact of telehealth on healthcare utilization by congestive heart failure patients. Dis Manage Health Outcomes. 14(3), pp. 163-169, 2006.

15. "Willem Einthoven," [Online]. Available: http://en.wikipedia. org/wiki/Willem_Einthoven [Accessed on April 22, 2014].

16. "Cardy 303 pico+," [Online]. Available: https://www.suzuken. co.jp/product/equipment/cardy303pico/index.html [Accessed on April 22, 2014].

17. Ishijima M: Monitoring of electrocardiograms in bed without utilizing body surface electrodes. IEEE Trans Biomed Eng. 40(6), pp. 593-594, 1993.

18. Watanabe K, Tasaki T, Nemoto T, Yamakoshi K and Chen W: Development of biometry system in the sleep by pillow cuff installed on the occiput. In: The 42nd Annual Conference of Japanese Society for Medical and Biological Engineering. Sapporo, 2003. [Online]. Available: http://www.jstage.jst.go.jp/article/ jsmbe2002/41/Supplement1/41_Supplement1_165/_article/ -char/ja/ (in Japanese).

19. Chen W, Zhu X, Nemoto T, Kanemitsu Y, Kitamura K, Yamakoshi K: Unconstrained detection of respiration rhythm and pulse rate with one under-pillow sensor during sleep. Med Biol Eng Comput. 43 (2), pp. 306-312, 2005.

20. Lim YG, Kim KK, Park K-S: ECG recording on a bed during 
sleep without direct skin-contact. IEEE Trans Biomed Eng. 54(4), pp. 718-725, 2007.

21. Lim YG, Kim KK, Park K-S: ECG measurement on a chair without conductive contact. IEEE Trans Biomed Eng. 53(5), pp. 956959, 2006.

22. Kwatra SC, Jain V: A new technique for monitoring heart signalspart I: instrumentation design. IEEE Trans Biomed Eng. 33(1), pp. 35-41, 1986.

23. Tamura T, Yoshimura T, Nakajima K, Miike H, Togawa T: Unconstrained heart-rate monitoring during bathing. Biomed Instrum Technol. 31(4), pp. 391-396, 1997.

24. Fujii M, Shutsuba H, Ueda T: Liquid jet electrode, liquid discharge electrode or their application in body surface potential probe. Japan Patent JPA2000-259200, 2982000.

25. Georgia Tech Wearable Motherboard ${ }^{\mathrm{TM}}$ : "The Intelligent Garment for the 21st Century," [Online]. Available: http://www. smartshirt.gatech.edu/ [Accessed on May 23, 2014].

26. Smartex S.r.l.: "Wearable Wellness System (WWS)," [Online]. Available: http://www.smartex.it/index.php/en/products/ wearable-wellness-system [Accessed on May 23, 2014].

27. Kim J, Kwon S, Park K: Preliminary study of use of embedded metal wires in brassiere as the electrodes for monitoring electrocardiogram in daily life. In International Conference on uHealthcare 2012. Kyeognju, Korea, 2012.

28. Patel P: "Electronic Skin Patch With Memory and Drug Delivery Capability Could Treat Parkinson's," 31 Mar 2014. [Online]. Available: http://spectrum.iee.org/tech-talk/biomedical/ devices/electronic-skin-patch-with-memory-and-drug-deliverycould-treat-parkinsons [Accessed on May 23, 2014].

29. Sorrel C: "Nike+ Heart-Rate Monitor On Sale June 1st," 16 2010. [Online]. Available: http://www.wired.com/2010/05/ nike-heart-rate-monitor-on-sale-june-1st/ [Accessed on May 23, 2014].

30. Jin Co., Ltd.: “JINS MEME," [Online]. Available: https://www. jins-jp.com/jinsmeme/en/product/ [Accessed on May 23, 2014].

31. Posokhov IN, Gant RH: "A brief history of Holter Monitoring," [Online]. Available: http://24h-monitoring.com/ecg/history. html [Accessed on May 23, 2014].

32. Chen W, Kawarada A, Togawa T: The development of ambulatory cordless sensor. In: The 67th Japan Medical Equipments. Okayama 1992.

33. Nihon Kohden Corp.: "Medical telemeter ZZ-100P," [Online]. Available: http://www.nihonkohden.co.jp/iryo/products/ monitor/01_bedside/zz100p.html\#doc_2 [Accessed on April 25, 2014].

34. Barrett P, Komatireddy R, Haaser S, Topol S, Sheard J, Encinas J, Fought A, XTopol E: Comparison of 24-hour Holter Monitoring with 14-day novel adhesive patch electrocardiographic monitoring. Am J Med. 127(1), p. 95, e11-95, e17, 2014.

35. Turakhia M, Hoang DD, Zimetbaum P, Miller JD, Froelicher VF, Kumar UN, Xu X, Yang F, Heidenreich PA: Diagnostic utility of a novel leadless arrhythmia monitoring device. Am J Cardiol. 112(4), pp. 520-524, 2013.

36. Rosenberg M, Samuel M, Thosani A, Zimetbaum P: Use of a noninvasive continuous monitoring device in the management of atrial fibrillation: a pilot study. Pacing Clin Electrophysiol. 36(3), pp. 328-333; 2012.

37. Schreiber D, Sattar A, Drigalla D, Higgins S: Ambulatory cardiac monitoring for discharged emergency department patients with possible cardiac arrhythmias. West J Emerg Med. 15(2), pp. 194 198, 2014.
38. Heart rate sensor "my Beat," [Online]. Available: http://www. uniontool.co.jp/en/product/sensor/index.html [Accessed on Oct $16,2014]$.

39. "Wireless Vital Sign Sensor" RF-ECG EK http://www. mmdevice.co.jp/english/goods_01.html [Accessed on October 16, 2014].

40. Meditech: "Merlin - one channel ECG event recorder built in a wristwatch," [Online]. Available: http://www.meditech.hu/ ecg-event-recorders/merlin-one-channel-ecg-event-recorderbuilt-in-a-wristwatch.html [Accessed on April 25, 2014].

41. “EP-201," [Online]. Available: http://www.parama-tech.com/ products/products_01.html [Accessed on April 26, 2014].

42. Cybernet Medical: "How MedStar Works," [Online]. Available: http://www.cybernetmedical.com/index.php/how-medstarworks [Accessed on April 26, 2014].

43. Viterion: "Infrastructure for today's telehealth professional," [Online]. Available: http://www.viterion.com/web_docs/Viterion \%20Telehealth\%20Network.pdf [Accessed on April 26, 2014].

44. Cardiocom: "Tiered platforms," [Online]. Available: http:// www.cardiocom.com/tiered_platforms.asp [Accessed on April 26, 2014].

45. Carematix: "CardioBlips," [Online]. Available: http://www. carematix.com/cardioblips.html [Accessed on April 26, 2014].

46. LifeWatch AG: "LifeStar ACT Ambulatory Cardiac Telemetry," [Online]. Available: http://www.lifewatch.com/ACT [Accessed on April 26, 2014].

47. Omron Healthcare Co. Ltd.: "MedicalLINK," [Online]. Available: http://www.ml.omron.co.jp/ht/pro/ [Accessed on April 26, 2014].

48. Tanita Corp.: "HealthPlanet," [Online]. Available: https://www. healthplanet.jp/ [Accessed on April 26, 2014].

49. Honeywell International Inc.: "Sentry Telemonitor - Total Solution," [Online]. Available: https://www.hommed.com/ lifestream-products/legacy-products-and-software/sentry-telemonitor/ [Accessed on April, 26].

50. Bosch Healthcare: "Health Buddy System," [Online]. Available: http://www.bosch-healthcare.com/en/us/products/health_ buddy/health_buddy.html [Accessed on April 26, 2014].

51. "BeClose," [Online]. Available: http://beclose.com/ [Accessed on April 26, 2014]

52. eNeighbor System, Healthsense [Online]. Available: http:// www.healthsense.com/ [Accessed on April 26, 2014].

53. iHealth Lab, Inc.: "Intel-GE Care Innovations ${ }^{\mathrm{TM}}$ and iHealth Partner to Make Home Health Monitoring Easier, Connect the Care Continuum to the Home," 155 2014. [Online]. Available: http://www.careinnovations.com/intel-ge-care-innovationsihealth-partner-make-home-health-monitoring-easierconnect-care-continuum-home/ [Accessed on June 6, 2014].

54. "Grand Care Systems," [Online]. Available: http://www. grandcare.com/ [Accessed on May 23, 2014].

55. “Philips' Telestation," [Online]. Available: http://www. telehealth.philips.com/solution_telestation.html/ [Accessed on October 6, 2014].

56. Omron WellnessLink http://www.wellnesslink.jp/p/ [Accessed on December 2, 2014].

57. Welch J, Moon J, McCombie S: Early detection of the deteriorating patient: the case for a multi-parameter patient-worn monitor. Biomed Instrum Technol. Suppl pp. 57-64, 2012.

58. Rutherford JJ: Wearable technology. IEEE Eng Med Biol Mag. 29(3), pp. 19-24, 2010.

59. Nippon Telegraph and Telephone Corporation: "Electrocardiography is achieved simply by wearing a piece of clothing that has 
textile electrodes combined with conductive polymer," 122 2013. [Online]. Available: http://www.ntt.co.jp/news2013/ 1302e/130212a.html [Accessed on May 23, 2014].

60. Paradiso R, De Rossi D: Advances in textile technologies for unobtrusive monitoring of vital parameters and movements. Conf Proc IEEE Eng Med Biol Soc. pp. 392-395, 2006.

61. Shim BS, Chen W, Doty C, Xu C, Kotov NA: Smart electronic yarns and wearable Fabrics for human biomonitoring made by carbon nanotube coating with polyelectrolytes. Nano Lett. 8(12), pp. 4151-4157, 2008.

62. Moore NC: "Nature, nanotechnology fuse in electric yarn that detects blood," 16 Dec 2008. [Online]. Available: http://ns. umich.edu/new/releases/6889 [Accessed on May 23, 2014].

63. "State of art Clevertex," 2008. [Online]. Available: http:// 109.2.243.201/Image/documents/State\%20of\%20the\%20art. pdf [Accessed on May 23, 2014].

64. Watts E: "High-Tech Tattoos Redefine Health Care Solutions," 7 June 2013. [Online]. Available: http://www.utexas.edu/know/ 2013/06/07/high-tech-tattoos-health-care-solutions/ [Accessed on May 23, 2014].

65. Son D, Lee J, Qiao S, Ghaffari R, Kim J, Lee J, Song C, Kim SJ, Lee DJ, Samuel Jun SW, Yang S, Park M, Shin J, Do K, Lee M, Kang K, Hwang CS, Lu N, Hyeon T, Kim D-H: "Multifunctional wearable devices for diagnosis and therapy of movement disorders," 303 2014. [Online]. Available: http://www.nature.com/ nnano/journal/vaop/ncurrent/full/nnano.2014.38.html [Accessed on June 6, 2014].

66. Asada HH, Shaltis P, Reisner A, Rhee S, Hutchinson RC: Mobile monitoring with wearable photoplethysmographic biosensors. IEEE Eng Med Biol Mag. 22(3) pp. 28-40, 2003.

67. Kwon H, Oh S, Kumar PS, Varadan VK: E-Bra system for women ECG measurement with GPRS communication, nanosensor, and motion artifact remove algorithm. In: Proc. SPIE 8548, Nanosystems in Engineering and Medicine. Incheon, Republic of Korea, 2012.

68. MTI Co., Ltd.: “Is your sleep satisfactory?," [Online]. Available: http://www.pc.karadafit.jp/ [Accessed on June 6, 2014].

69. Tamura T, Maeda Y, Sekine M, Yoshida M: Wearable photoplethysmographic sensors-past and present. Electronics. 3(2), pp. 282-302, 2014.

70. Taylor B: "26 Fitness Trackers Ranked from Worst to First," 91 2014. [Online]. Available: http://time.com/516/26-fitnesstrackers-ranked-from-worst-to-first/ [Accessed on June 6, 2014].

71. Sazonova N, Browning R, Sazonov E: Accurate prediction of energy expenditure using a shoe-based activity monitor. Med Sci Sports Exerc. 43(7), pp. 1312-1321, 2011.

72. Cole P, Lemura LM, Klinger TA, Strohecker K, McConnell T. Measuring energy expenditure in cardiac patients using the Body Media Armband versus indirect calorimetry. A validation study. J Sports Med Phys Fitness. 44(3), pp. 262-271, 2004.

73. BodyMedia: "Introducing the BodyMedia SenseWear System," [Online]. Available: http://sensewear.bodymedia.com/ [Accessed on June 6, 2014].

74. "Misfit Shine activity tracker" [Online]. Available: http://misfit. com/media [Accessed on October 16, 2014].

75. Dai J, Bai X, Yang Z, Shen Z, Xuan D: Mobile phone-based pervasive fall detection. Pers Ubiquit Comput. 14(7), pp. 633-643, 2010.

76. "A wellness mobile phone for pulse, body fat, breath gas, calories measurement," 210 2007. [Online]. Available: http://www. itmedia.co.jp/mobile/articles/0710/02/news036.html [Accessed on April 4, 2014].
77. Abe T, Chen W, Togawa T, Maeda T, Arai M: Development of a mobile phone-based blood pressure monitor. In: The 46th Annual Conference of Japanese Society for Medical and Biological Engineering. Sendai, Japan, 2007. [Online]. Available https://www. jstage.jst.go.jp/browse/jsmbe/45/Supplement1/_contents/ -char/ja/ [Accessed on July 1, 2014].

78. Fitzpatrick T: "Ultrasound imaging now possible with a smartphone," 204 2009. [Online]. Available: http://news.wustl.edu/ news/Pages/13928.aspx [Accessed on April 23, 2014].

79. Kailas A, Chong C-C, Watanabe F: From mobile phones to personal wellness dashboards. IEEE Pulse. 1(1), pp. 57-63, 2010.

80. Tamura T, Togawa T, Murata M: A bed temperature monitoring system for assessing body movement during sleep. Clin Phys Physiol Meas. 9(2), pp. 139-145, 1988.

81. Togawa T, Mizukami H, Tamura T: Physiological monitoring techniques for home health care. Biomed Sci Instrum. 28, pp. 105-110, 1992.

82. Ishijima M: Cardiopulmonary monitoring by textile electrodes without subject-awareness of being monitored. Med Biol Eng Comput. 35(6), pp. 685-690, 1997.

83. Chen W, Zhu X, Nemoto T, Kanemitsu Y, Kitamura K, Yamakoshi Y: Unconstrained detection of respiration rhythm and pulse rate with one under-pillow sensor during sleep. Med Biol Eng Comput. 43(2), pp. 306-312, 2005.

84. Omron Healthcare Corp.: "New Omron Sleep Monitoring Devices Unveiled," Omron Healthcare Corp., 292 2012. [Online]. Available: http://www.omron.co.jp/press/2012/02/h0229_2. html [Accessed on April 23, 2014].

85. Staderini E: UWB radars in medicine, IEEE Aerosp Electron Syst Mag. 17(1), pp. 13-18, 2002.

86. Massagram W, Lubecke V, Host-Madsen A, Boric-Lubecke O: Assessment of heart rate variability and respiratory sinus arrhythmia via Doppler radar. IEEE Trans Microw Theory Tech. 57(10), pp. 2542-2549, 2009.

87. Chong J, Lai Y, Xu Y, Gunawan E, Chua C, Maskooki A, Guan Y, Low K, Soh C, Poh C: Wireless sensing of human respiratory parameters by low-power ultra-wideband impulse radio radar. IEEE Trans Instrum Meas. 60(3), pp. 928-938, 2011.

88. Tanita Corp.: “Sleep Scan SL-504," [Online]. Available: http:// www.tanita.co.jp/shop/g/_TSL504WH/ [Accessed on June 6, 2014].

89. Denso Co.: Sleep EYE GD700 [Online]. Available: http://www. denso.co.jp/ja/products/consumer/healthcare/index.html [Accessed on December 22, 2014].

90. Suzuken Co., Ltd.: "Japan's first! Medical equipment that can check sleep apnea unrestrainedly (Japanese)," Suzuken Co., Ltd., [Online]. Available: https://www.suzuken.co.jp/company/ news/2007/07-08-22.html [Accessed on June 6, 2014].

91. Agatsuma T, Fujimoto K, Komatsu Y, Urushihata K, T. Honda T, Tsukahara T, Nomiyama T: A novel device (SD-101) with high accuracy for screening sleep apnoea-hypopnoea syndrome. Respirology. 14(8), pp. 1143-1150, 2009.

92. Fujii M, Dema H, Ueda T: Liquid jet electrode and surface potential detector. Japan Patent JP2002-65625A, 532002.

93. Ishijima M: Novel approaches to monitor vital signs without awareness of being monitored. IFMBE Proceedings. 14(6), pp. 4039-4042, 2007. Med Biol Eng Comput. 35(6), pp. 685690, 1997.

94. Okada M: Iryukiki ga ichiban wakaru (The most understanding medical devices)" Gijyutsuhyoron Co. p. 205, 2009 (in Japanese).

95. Yamakoshi K, Kuroda M, Tanaka S, Yamaguchi I, Kawarada A: 
Non-conscious and automatic acquisition of body and excreta weight together with ballistocardiogram in a lavatory. Engineering in Medicine and Biology Society, 1996. Proceedings of the 18th Annual International Conference of the IEEE. 1, pp. 67-68, 1996.

96. Nippon Avionics Co., Ltd.: "Noncontact thermal mirror," [Online]. Available: http://www.infrared.avio.co.jp/jp/products/ thermo-mirror/ [Accessed on June 6, 2014].

97. "When your carpet calls your doctor," [Online]. Available: http:// www.economist.com/node/15868133. [Accessed on June 6, 2014].

98. Kobayashi T, Chen W: Meal measuring device and analysis system. Japan Patent JP3588031, 20082004.

99. Aleksandrowicz A, Leonhardt S: Wireless and non-contact ECG measurement system- the "Aachen Smart Chair" Acta Polytechnica. 47(4-5), pp. 68-71, 2007.

100. Futatsuyama K, Mitsumoto N, Kawachi T, Nakagawa T: Noise robust optical sensor for driver's vital signs. Denso Tech Rev. 17, pp. 119-125, 2012.

101. Tamura T, Home geriatric physiological measurements. Physiol Meas. 33(10), pp. R47-65, 2012.

102. WHO: A Long-Term Care Futures Tool-Kit, Pilot edition, WHO, 2002.

103. Tamura T, Maeno S, Hattori T, Kimura Y, Kimura Y, Yoshida M, Minato K: Assessment of participant compliance with a Webbased home healthcare system for promoting special health checkups. Biocybern Biomed Eng. 34(1), pp. 63-69, 2014.

104. Medtronic, Inc.: "Small, Wireless Monitor Provides Long-Term Remote Monitoring to Help Physicians Diagnose and Monitor Irregular Heartbeats," 192 2014. [Online]. Available: http:// newsroom.medtronic.com/phoenix.zhtml?c=251324\&p=irolnewsArticle \&ID=1901216\&highlight=linq [Accessed on June 6, 2014].

105. Kim KK, Lim YG, Kim JS, Park KS: Effect of missing RR-interval data on heart rate variability analysis in the time domain. Physiol Meas. 28, pp. 1485-1494, 2007.

106. Kim KK, Kim JS, Lim YG, Park KS: The effect of missing RR-interval data on heart rate variability analysis in the frequency domain. Physiol Meas. 30, pp. 1039-1050, 2009.

107. Kim KK, Baek HJ, Lim YG, Park KS: Effect of missing RR-interval data on nonlinear heart rate variability analysis. Comput Methods Programs Biomed. 106(3), pp. 210-218, 2012.

108. Tseng Y-H, He Y, Lakshmanan S, Yang C, Chen W, Que L: Optical and thermal response of single-walled carbon nanotube-copper sulfide nanoparticle hybrid nanomaterials. Nanotechnology. 23(45), pp. 455708, 2012.

109. WiTricity Corporation: "Wireless Electricity Delivered Over Distance," [Online]. Available: http://www.witricity.com/ [Accessed on June 6, 2014].

110. IEEE 802.15 WPAN Task Group 7 (TG7), "Visible Light Communication," 255 2014. [Online]. Available: http://www. ieee802.org/15/pub/TG7.html [Accessed on June 6, 2014].

\section{Toshiyo Tamura}

Dr. Toshiyo Tamura received Ph.D. from Tokyo Medical and Dental University in 1980. He is currently a Distinguished Professor, School of Biomedical Engineering, Osaka Electro-Communication University, Japan. He also holds several adjunct positions in universities in Japan and Sin-

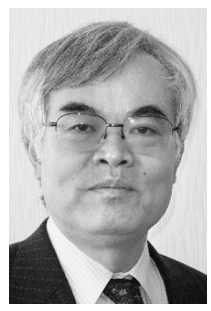
gapore. His research interests include biomedical instrumentation, biosignal processing, telemedicine telecare, home care technology and rehabilitation engineering. His research has resulted in over 120 English reviewed articles. He has served as a chair of IEEE/EMBS Tokyo Chapter in 1996-2000, and the Asian Pacific representative for the EMBS from 2000 to 2004. He was a president of Japanese Society of Medical Electronics and Biological Engineering in 2010-2012 and a president of Japanese Society of Life Support Technology in 20092011.

\section{Wenxi Chen}

Wenxi CHEN received Bachelor and Master Degrees in Biomedical Engineering from Zhejiang University, China in 1983 and 1986, respectively. He received Ph.D. degree in biomedical instrumentation from Institute of Biomaterials and Bioengineering, Tokyo Medical and Dental Universi-

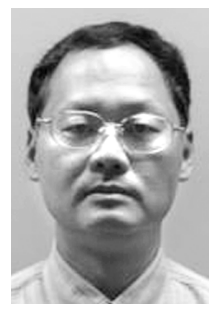
ty, Japan in 2001. He is currently a professor in Biomedical Information Technology Lab in the University of Aizu. He has participated more than 10 major R\&D projects funded by Japanese government ministries, Fukushima prefecture and industries since 1998. These activities produced more than 30 patents (20 registered) and more than 160 papers. Some academic outcomes have been commercialized or are in progress. His current research interests focus on developing diversified modalities to detect physiological signal under daily life environment, and on performing comprehensive interpretation of multifarious longterm data to reveal statistical links and causalities among diseases as well as how they interact with various factors in temporal/spatial domains. 